Ann. Biol. anim. Bioch. Biophys., I967, 7 (I), 63-71.

\title{
INFLUENCE DES SULFITES SUR LA PROTÉOLYSE : ETUDE IN VITRO
}

\author{
P. MOUTONNET \\ avec la collaboration technique de W. LOISEL \\ Laboratoire d'Étude des Qualités biologiques des Aliments de l'Homme, \\ Centre national de Recherches zootechniques, 78-Jouy-en-Josas
}

SOMMAIRE

Même à dose élevée, les sulfites n'ont aucune influence sur l'hydrolyse des protéines du lait écrémé en poudre, de la gélatine et du gluten par la pepsine, la trypsine et la peptidase.

En revanche, ils retardent l'hydrolyse enzymatique des protéines du blanc d'œuf cru et, dans une moindre mesure, du blanc d'oeuf cuit. Il est possible que cet effet soit dû à une combinaison des sulfites avec les acides aminés soufrés, particulièrement abondants dans les protéines de l'œuf.

\section{INTRODUCTION}

L'anhydride sulfureux et quelques-uns des sels qui en dérivent (métabisulfite de potassium, sulfite de sodium) figurent parmi les antiseptiques les plus anciennement employés en technologie alimentaire (DUPUY, I959; CAUSERET, I960; CrUZAN et al., r965) : on connaît en particulier leurs utilisations en œnologie, en cidrerie, en brasserie, dans l'industrie des jus de fruits, dans le traitement de certains fruits et légumes en vue de leur conservation, etc.; on sait également que le métabisulfite de sodium peut être employé dans la préparation des ensilages.

Cependant, les effets des sulfites sur l'organisme du consommateur restent insuffisamment connus. Sans insister ici sur les aspects proprement toxicologiques de la question - aspects qui ont été envisagés par CluZAN et al. (I965) -, nous voudrions souligner qu'on ne s'est guère préoccupé jusqu'ici des effets éventuels 
de ces substances sur certaines fonctions physiologiques. On peut se demander en particulier si les sulfites, qui exercent un effet inhibiteur sur diverses réactions enzymatiques (DUPUY, 1959), ne risquent pas d'entraver l'action des enzymes digestives.

Il nous a donc paru intéressant d'aborder l'étude de ce dernier problème.

\section{MATÉRIEL ET MÉTHODES}

Dans ce mémoire, nous présentons les résultats d'une série d'expériences portant sur l'activité, in vitro, de diverses enzymes purifiées (pepsine, trypsine, peptidase) en présence ou en l'absence de sulfites. Différents types de substrats ont été utilisés :

- d'origine animale : lait en poudre écrémé, gélatine, blanc d'œuf;

- d'origine végétale : gluten.

\section{Caractéristiques des substrats utilisés}

L'origine et les caractérístiques des substrats utilisés sont indiquées dans le tableau 1 .

TABLEAU I

Origine et caractéristiques des différents substrats

\begin{tabular}{|c|c|c|c|}
\hline Substrats & Traitements & $\begin{array}{c}\text { Teneur en protéines } \\
(\%)\end{array}$ & $\begin{array}{c}\text { Teneur en eau } \\
(\%)\end{array}$ \\
\hline Poudre de lait écrémé...... & Atomisation & $37,7(\mathrm{~N} \times 6,39)$ & - \\
\hline Gélatine (N.B.C.) . ........ & - & $99,3(\mathrm{~N} \times 6,25)$ & - \\
\hline 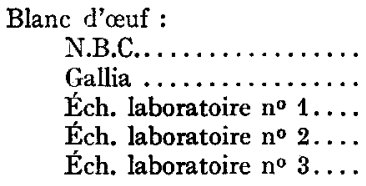 & $\begin{array}{l}\text { Atomisation } \\
\text { Atomisation } \\
\text { Séchage à } 50^{\circ} \mathrm{C}\left({ }^{1}\right) \\
\text { Lyophilisation }\left({ }^{\mathbf{2}}\right) \\
\text { Cuisson et lyophilisation }\left(^{2}\right)\end{array}$ & $\begin{array}{l}78,7(\mathrm{~N} \times 6,25) \\
80,7(\mathrm{~N} \times 6,25) \\
86,6(\mathrm{~N} \times 6,25) \\
81,2(\mathrm{~N} \times 6,25) \\
84,0(\mathrm{~N} \times 6,25)\end{array}$ & $\begin{array}{l}6,0 \\
\mathbf{6 , 3} \\
2,9 \\
\text { traces } \\
\text { traces }\end{array}$ \\
\hline Gluten (N.B.C.) $\ldots \ldots \ldots \ldots$ & - & $73,0(\mathrm{~N} \times 5,70)$ & - \\
\hline
\end{tabular}

() Le blanc d'œuf est traité par l'alcool à $95^{\circ}$ (2 volumes d'alcool pour 1 volume de blanc d'œuf). Le coagulum est filtré sur büchner, séché sous vide à l'étuve à $50^{\circ} \mathrm{C}$, puis pulvérisé au broyeur à bille.

${ }^{(2)}$ Le blanc d'œuf est battu en neige, puis on prépare deux échantillons :

$\mathrm{n}^{\circ} 2$ - échantillon " cru " : lyophilisé immédiatement.

$n^{0} 3$ - échantillon "cuit ": le blanc battu est passé au bain-marie à $100^{\circ} \mathrm{C}$ pendant 5 minutes avant la lyophilisation.

Pour les deux types de préparation, la lyophilisation est réalisée sur plateaux pendant $\mathbf{2} 4$ heures.

Les produits secs sont mis en poudre au mixer.

\section{Techniques d'hydrolyse}

L'hydrolyse porte sur des échantillons de $2 \mathrm{~g}$ (poudre de lait) ou de $\mathrm{g}$ (tous les autres substrats). Ces prises sont introduites dans des matras et on ajoute à une partie d'entre elles :

- soit du métabisulfite de potassium à la dose de 50 ou de $25^{\circ} \mathrm{mg}$ par gramme de poudre ; 
- soit du sulfite de sodium à la dose de roo mg par gramme de poudre (1).

Deux hydrolyses successives sont pratiquées sur chaque échantillon, les matras étant plongés dans un bain-marie à $4^{\circ} \mathrm{C} \pm 0,5^{\circ} \mathrm{C}$ avec agitation permanente (système électromagnétique) :

I. Hydrolyse pepsique de 30 minutes portant sur les I ou $2 \mathrm{~g}$ de substrat dissous dans $25 \mathrm{ml}$ d'HCl $\mathrm{N} / \mathrm{I} 0$ contenant la pepsine $\left({ }^{2}\right)$ à 0,20 p. I00 (pH env. $\left.\mathrm{r}, 8\right)$.

2. Hydrolyse trypsique de 6 heures, après neutralisation par la soude $0,2 \mathrm{~N}$. En ce qui concerne ce deuxième stade, il y a deux séries différentes :

— avec $15 \mathrm{ml}$ de tampon phosphaté o, I $\mathrm{M}$ contenant la trypsine (3) à o,25 p. Ioo ;

- avec i $5 \mathrm{ml}$ de tampon phosphaté $0,1 \mathrm{M}$ contenant la trypsine à $0,25 \mathrm{p}$. Ioo et la peptidase $\left(^{4}\right)$ à 0,40 p. IO0.

Pour chaque prélèvement - au temps $t$ - les hydrolysats sont soumis à une ébullition de I 5 minutes au bain-marie, pour assurer à la fois l'arrêt de l'hydrolyse et la dénaturation des protéines restantes. Les prélèvements sont ensuite centrifugés ( 5 minutes à $4000 \mathrm{~g}$ ).

\section{Dosage de l'azote aniné}

L'azote aminé total est dosé par la méthode de Michel (I960) (réaction à la ninhydrine, puis microdiffusion d'ammoniac dans des cellules de Conway).

Il est à signaler que l'utilisation d'autres techniques de dosage du $\mathrm{N}\left(\mathrm{NH}_{2}\right)$ avait d'abord été envisagée sans succès :

I. Par spectrophotométrie à $280 \mathrm{~m} \mu$ où tryptophane, cystéine, phénylalanine absorbent (Lowy et $a l$. ., 1958) : en principe, la mesure de la densité optique à cette longueur d'onde permet donc de suivre la cinétique d'hydrolyse enzymatique. Mais, dans le cas présent, le sulfite réagit avec la cystéine pour donner finalement un complexe qui absorbe à $267 \mathrm{~m} \mu$ (DUPUY, I959). D'où impossibilité totale de mesure.

2. Par méthode manométrique de Van Slyke à l'acide nitreux (Van Slyke, I9II). Au cours de la réaction, l'anhydride sulfureux fixé est libéré avec l'azote et interfère dans le dosage.

\section{RÉSULTATS}

Les résultats réunis dans les tableaux 2 et 3 sont exprimés en milligrammes de $\mathrm{N}\left(\mathrm{NH}_{2}\right)$ par gramme de protéines.

I. On voit que, dans le cas du lait, de la gélatine et du gluten, les différences observées entre les échantillons hydrolysés en présence de sulfite et les échantillons témoins sont généralement minimes : le calcul statistique, par la méthode du $t$ de Student, montre qu'elles ne sont jamais significatives. On peut donc considérer que, dans les conditions d'expérience adoptées, les sulfites n'ont pas d'effet sur la protéolyse des trois substrats.

2. Il n'en est pas de même dans le cas du blanc d'ceuf, dont l'azote aminé est libéré plus lentement lorsque l'hydrolyse est réalisée en présence de sulfite.

Avec les deux échantillons du commerce (N.B.C., Gallia) et le blanc d'œuf cru lyophilisé (échantillon laboratoire $n^{\circ} 2$ ), les différences entre produit ayant subi

( ${ }^{\mathrm{I}}$ ) Rapportées au gramme de protéines, les doses de $\mathrm{SO}_{2}$ utilisées atteignent en moyenne respectivement $65 \mathrm{mg}$ et $360 \mathrm{mg}$ pour le métabisulfite de potassium, I30 $\mathrm{mg}$ pour le sulfite de sodium. Or, dans la ration d'un homme adulte ingérant $80 \mathrm{~g}$ de protéines et consommant I 1 de vin sulfité à la dose licite maximum (350 $\mathrm{mg}$ de $\mathrm{SO}_{2}$ par litre), la correspondance serait de $4,4 \mathrm{mg}$ de $\mathrm{SO}_{2}$ par gramme de protéines. Quelles que soient les réserves que l'on peut opposer à cette comparaison, les doses de $\mathrm{SO}_{2}$ utilisées dans notre expérience apparaissent donc comme élevées.

(2) Pepsine $1 / 10000$ Difco.

(3) Trypsine $1 / 250$ Difco.

(') Peptidase - "Porcine intestine - C Grade» - Calbiochem.

Annales de Biologie animale. - 1967. 
l'action du sulfite et produit ne 1'ayant pas connue sont, à tous les stades de prélèvement, significatives au seuil de o,or.

Avec 1'albumine d'œuf obtenue par précipitation à 1'alcool à $95^{\circ}$ et séchage ménagé à l'étuve (échantillon laboratoire $\mathrm{n}^{0} \mathrm{I}$ ), et le blanc d'œuf cuit lyophilisé, les différences entre produit hydrolysé en présence de sulfite et produit témoin sont :

- significatives au seuil de $P=0,05$ pour les prélèvements $P_{1}$ (fin de l'attaque pepsique), $\mathrm{T}_{1}$ (5 minutes d'hydrolyse trypsique) et $\mathrm{T}_{2}$ (30 minutes d'hydrolyse trypsique) ;

- non significatives pour la suite.

Pour savoir à quel stade de la protéolyse se situe l'action du sulfite, une série d'hydrolyses sur le blanc d'œuf cru lyophilisé (échantillon laboratoire $n^{\circ}$ 2) a été réalisée en introduisant le sulfite :

- soit avant 1'attaque pepsique ;

- soit aussitôt après, en même temps que la trypsine et la peptidase.

La figure I montre que l'action du sulfite se situe au niveau de 1'attaque pep-

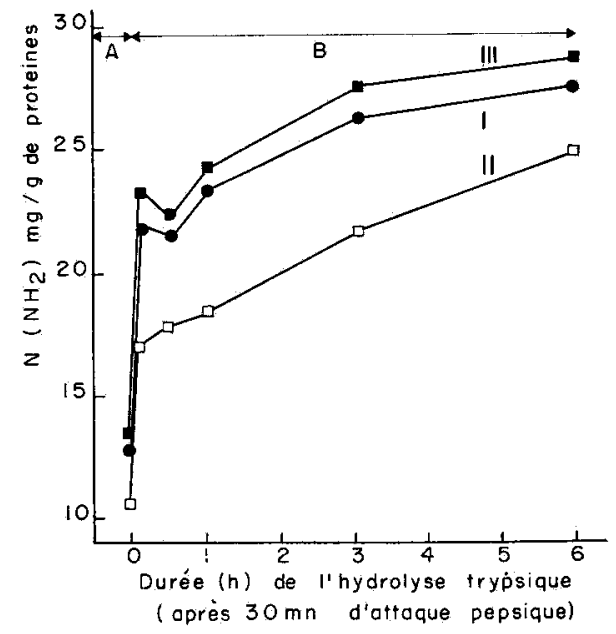

FIG. I. - Effets du sulfite de sodium sur l'attaque pepsique

et sur l'hydrolyse "trypsine-peptidase "du blanc d'auf cru lyophilise.

I. Échantillon témoin.

II. Échantillon additionné de sulfite de sodium (I oo $\mathrm{mg}$ par g de substrat) avant l'attaque pepsique.

III. Échantillon additionné de sulfite de sodium (à la même dose) après l'attaque pepsique. (Période $\mathrm{A}$ : attaque pepsique. Période B : hydrolyse trypsique).

sique. A chaque stade des prélèvements, les différences observées entre échantillon témoin et échantillon additionné de sulfite dès le début de l'attaque pepsique sont significatives au seuil de $\mathrm{P}=0, \mathrm{or}$. Mais elles ne le sont pas entre échantillon témoin et échantillon additionné de sulfite après l'attaque pepsique.

Le retard de l'hydrolyse trypsique observé en présence de sulfite semble résulter uniquement d'une attaque pepsique moins poussée. 


\section{DISCUSSION}

I. On peut s'étonner du faible pourcentage d'hydrolyse: après 6 heures d'attaque par la trypsine, l'azote aminé libéré correspond approximativement à $20 \mathrm{p}$. Ioo de l'azote total dans le cas du lait, de la gélatine et du blanc d'œuf, à 30 p. Ioo dans le cas du gluten.

Quelques essais, effectués sur le lait, ont montré qu'il était possible d'améliorer ce pourcentage en prolongeant la durée de l'attaque trypsique : mais, après 25 heures, il n'atteignait encore que $32 \mathrm{p}$. Ioo $\left(^{1}\right)$. Compte tenu du fait que nous nous intéressions surtout à la comparaison de vitesses d'hydrolyse, cette amélioration ne nous a pas semblé suffisante pour justifier le prolongement systématique de l'attaque trypsique au-delà de 6 heures.

L'utilisation de la peptidase accroît la vitesse de l'hydrolyse trypsique du lait : en 6 heures, elle fait passer le pourcentage d'azote aminé libéré à 26 p. Ioo (au lieu de $20 \mathrm{p}$. IOO). Mais, elle est apparemment sans action lorsque le substrat est la gélatine, le blanc d'œuf ou le gluten.

D'autre part, nous avons constaté que l'addition d'ions $\mathrm{Mn}^{++}$est sans effet avec tous les substrats.

En fait, le contraste entre la lenteur de l'hydrolyse trypsique dans nos essais et sa plus grande rapidité dans ceux d'autres auteurs est probablement plus apparent que réel. En effet, la méthode de Miches pour le dosage de l'azote aminé ne rend compte que de $\mathrm{N}\left(\mathrm{NH}_{2}\right)$ libre. D'autres méthodes, comme celle de VAN SI,YKE (non utilisée dans ce travail pour des raisons qui ont été indiquées plus haut), dosent 1'azote de petits peptides en même temps que l'azote aminé libre.

Comme l'a observé MrChÉ, (I960), la plus ou moins grande spécificité des méthodes employées pour le dosage de l'azote aminé peut expliquer bien des divergences expérimentales. Les résultats que nous avons obtenus en déterminant l'azote aminé par plusieurs méthodes au cours de 1'hydrolyse trypsique de la poudre de lait en fournissent une nouvelle preuve (tabl. 4). Bien que l'allure générale des différentes courbes obtenues soit la même, on voit que la signification d'une valeur expérimentale isolée est très limitée. Le principal intérêt des dosages d'azote aminé dans un liquide d'hydrolyse est de permettre de suivre le progrès de cette hydrolyse au cours du temps, et surtout de comparer la cinétique du phénomène dans différentes conditions expérimentales : ce qui était le cas ici.

Il y a lieu enfin de souligner que les acides aminés libérés ont probablement une action inhibante sur l'hydrolyse enzymatique. On améliorerait donc peut-être les résultats en réalisant une dialyse continue des produits formés (MAURoN et al., I955). Mais il faudrait veiller alors à maintenir constante la concentration du milieu en sulfites.

2. Il n'est pas sans intérêt de souligner que la cinétique de la protéolyse est sensiblement la même pour tous les substrats utilisés, à l'exception du gluten (tabl. 2 et 3). Dès la fin de l'attaque pepsique, la quantité de $\mathrm{N}\left(\mathrm{NH}_{2}\right)$ libérée à partir de ce substrat est particulièrement élevée ; 1'hydrolyse trypsique est plus rapide aussi que celle du lait, de la gélatine ou du blanc d'œuf.

(1) Pour les hydrolyses de longue durée, une couche de toluène a permis d'éviter contaminations et fermentations à l'intérieur des matras. 
P. MOUTONNET




Il serait intéressant de développer l'étude de cet aspect du problème et de chercher quelle peut être sa portée pratique.

TABLEAU 3

Azote aminé libéré au cours de l'hydrolyse "pepsine-trypsine + peptidase de différents substrats, en l'absence ou en présence de salfiles.

(Chaque valeur corespond à la moyenne de 4 déterminations)

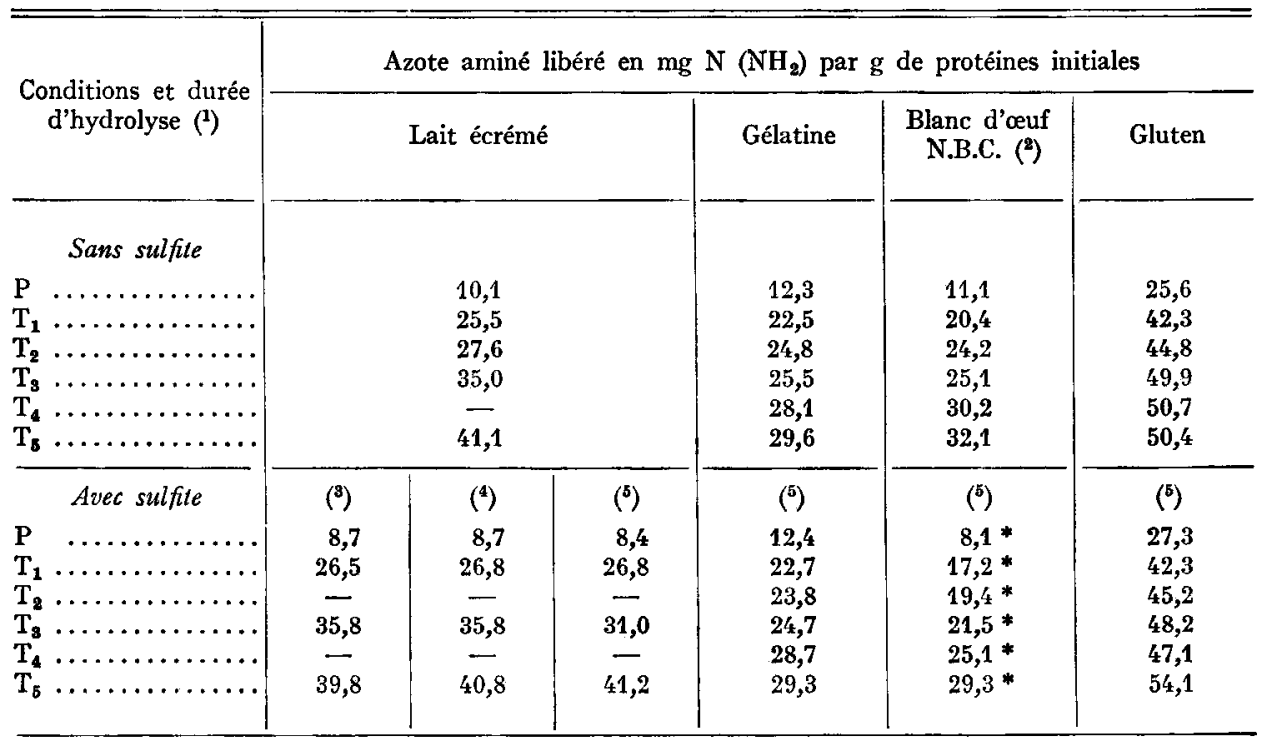

(1) $\mathrm{P}$ : après attaque pepsique $(30 \mathrm{mn})$;

$\mathrm{T}_{1}, \mathrm{~T}_{2}, \mathrm{~T}_{3}, \mathrm{~T}_{4}, \mathrm{~T}_{5}$ : après attaque pepsique $(30 \mathrm{mn})$ suivie d'une hydrolyse trypsique de $5 \mathrm{mn}, 30 \mathrm{mn}$, $1 \mathrm{~h}, 3 \mathrm{~h}, 6 \mathrm{~h}$.

${ }^{(2)}$ Un astérisque signifie que la différence entre les résultats obtenus en l'absence et en présence de sulfite est significative, au seuil de 0,01 .

(3) $50 \mathrm{mg}$ de métabisulfite de potassium par g de substrat.

(4) $250 \mathrm{mg}$ de métabisulfite de potassium par $\mathrm{g}$ de substrat.

(5) $100 \mathrm{mg}$ de sulfite de sodium par $\mathrm{g}$ de substrat.

3. S'il n'est pas possible d'expliquer de manière certaine pourquoi les sulfites retardent 1'hydrolyse des protéines d'œuf, mais non celle des autres substrats utilisés, on peut néanmoins émettre une hypothèse.

Une différence essentielle entre les protéines du blanc d'œuf et les autres substrats est la teneur plus élevée des premières en acides aminés soufrés :

Acides aminés ( $\mathrm{g}$ pour roo $\mathrm{g}$ d'azote)

Cystine Méthionine Total

\begin{tabular}{|c|c|c|c|}
\hline Lait $\ldots \ldots \ldots \ldots \ldots$ & 0,9 & 2,6 & 3,5 \\
\hline Blanc d'œuf.... . & 2,7 & 3,8 & 6,5 \\
\hline Gélatine ........ & $0, \mathbf{I}$ & 0,8 & 0,9 \\
\hline Gluten $\ldots \ldots \ldots \ldots$ & $2, \mathrm{I}$ & $\mathrm{I}, 4$ & 3,5 \\
\hline
\end{tabular}


Elles sont donc particulièrement riches en liaison $\mathrm{S}-\mathrm{S}$ ou $-\mathrm{SH}$ qui réagissent avec $\mathrm{SO}_{3}{ }^{--}$pour donner $-\mathrm{S}-\mathrm{SO}_{2}$.

TABLEAU 4

Dosage de l'azote aminé par différentes méthodes, après hydrolyse pepsique et trypsique du lait écrémé en poudre

\begin{tabular}{|c|c|c|c|}
\hline \multirow[b]{2}{*}{$\begin{array}{l}\text { Durée de l'hydrolyse: } \\
\text { — pepsique : } \mathrm{P} \\
\text { - trypsique : } \mathrm{T}\end{array}$} & \multicolumn{3}{|c|}{ Dosage de l'azote aminé selon: } \\
\hline & $\begin{array}{l}\text { Méthode MICHEL } \\
\text { sur échant. déprotéinés } \\
\text { par ébullition suivie de } \\
\text { centrifugation. } \\
\text { (mg } \mathrm{N}\left(\mathrm{NH}_{2}\right) \text { par } \mathrm{g} \text { de } \\
\text { protéines initiales) }\end{array}$ & $\begin{array}{c}\text { Méthode MrcheL } \\
\text { sur échant. déprotéinés } \\
\text { à l'alcool à 950 } \\
\text { (mg N }\left(\mathrm{NH}_{2}\right) \text { par g de } \\
\text { protéines initiales) }\end{array}$ & $\begin{array}{c}\text { Méthode VaN SLYKe à } \\
\text { l'acide nitreux (mg N } \\
\left(\mathrm{NH}_{2}\right) \text { par g de protéines } \\
\text { initiales) }\end{array}$ \\
\hline $\mathrm{P}=30 \mathrm{mn} \ldots$ & 9,7 & 5,3 & - \\
\hline $\mathrm{T}_{1}=5 \mathrm{mn} \ldots \ldots \ldots$ & 23,7 & 11,7 & 53,0 \\
\hline $\mathrm{T}_{2}=30 \mathrm{mn} \ldots \ldots \ldots$ & 24,7 & 17,7 & 53,4 \\
\hline $\mathrm{T}_{3}=1 \mathrm{~h} \ldots \ldots \ldots \ldots$ & 27,6 & 17,2 & 56,8 \\
\hline $\mathrm{T}_{4}=3 \mathrm{~h} \ldots \ldots \ldots \ldots$ & 30,4 & 21,5 & 58,8 \\
\hline $\mathrm{T}_{5}=6 \mathrm{~h} \ldots \ldots \ldots$ & 33,0 & 21,7 & 62,6 \\
\hline
\end{tabular}

$N . B-$ Ces valeurs correspondent à la moyenne obtenue à partir de $t_{\mathfrak{t}}$ expériences successives.

Ce serait la liaison-S-S $\mathrm{SO}_{2}$ qui gênerait l'évolution normale de 1'hydrolyse enzymatique du blanc d'œuf $c r u$. Par contre, dans le blanc cuit ou dénaturé à l'alcool, les liaisons $\mathbf{S}-\mathbf{S}$ ou $-\mathbf{S H}$ sont dégradées partiellement et n'entreraient plus en réaction avec $\mathrm{SO}_{3}^{--}$: d'où une cinétique enzymatique différente.

C'est d'ailleurs une hypothèse du même type (formation de complexes entre sulfite et groupements - SH) que DuPUy (I959) émet pour expliquer l'inhibition par l'acide sulfureux de l'oxydation de 1'éthanol par Acetobacter rancens.

Reçu pour publication en octobre $\mathbf{1 9 6 6 .}$

\section{REMERCIEMENTS}

Nous adressons nos vifs remerciements à MM. G. Fauconneau, R. Pion et M.-C. Michel pour leurs précieux conseils.

\section{SUMMARY}

INFLUENCE OF SULPHITES ON PROTEOLYSIS : STUDIES \& IN VITRO \#

The author studied the influence of certain sulphites on the hydrolysis of different substrates by pepsin, trypsin and peptidase. Potassium metabisulphite was added as $5 \circ$ or $25^{\circ}$, and sodium sulphite as Ioo $\mathrm{mg}$ per $\mathrm{g}$ substrate. Free amino nitrogen was estimated by the method of MiCHEL (Reaction with ninhydrin then micro-diffusion of ammonia in the cells of CoNway.) 
In these conditions the sulphites did not have any effect on the rate on hydrolysis of the proteins of skimmed milk powder, gelatin or gluten. They did slow down the hydrolysis of egg white, particularly if raw ; with cooked egg white the effect was less marked. It seemed that the retarding action of the sulphites exerted itself on the development of the attack by pepsin ; the effect was not seen if the sulphite was added to the egg white only at the start of trypsin hydrolysis.

It is possible that the particular behaviour of egg white proteins may be due to their richness in sulphur amino acids. In effect, the S-S or -SH bonds react with $\mathrm{SO}_{3}-$ to give $-\mathrm{S}^{-} \mathrm{SO}_{2}$ bonds which can hinder the development of hydrolysis.

\section{RÉFÉRENCES BIBLIOGRAPHIQUES}

CAUSERET J., I960. Répercussions hygiéniques et nutritionnelles de quelques traitements de stabilisation des boissons. Diét. Nutrit., 4I-5I.

Cluzan R., Causeret J., Hugot D., I965. Le métabisulfite de potassium : étude de toxicité à long terme sur le Rat. Ann. Biol. anim., Bioch., Biophys., 5, 267-281.

Dupuy P., 1959. L'inhibition par l'acide sulfureux de l'oxydation de l'éthanol par Acelobacter rancens. Ann. Technol. agric., 8, $233^{-28} 3$ et $337-376$.

Lowy R., Brigand L., TrÉmolitères J., 1958. Intervention de divers types d'amidon dans la vitesse de digestion des protéines. Ann. Nutrit. Alim., 12, 51-59.

Miches M. C., I960. Technique de microdosage de l'azote $\alpha$-aminé : dosage de l'azote aminé dans quelques liquides biologiques. Aminoacides, peptides, protéines. Cahier no 4, 63-71, A. E. C., Commentry.

VAN Slyke D., IgII. J. biol. Chem., 4, 3. 